\title{
Das Leben mit einem fremden Organ
}

\author{
Lungentransplantationen (LTX) sind oftmals die einzig \\ verbleibende Behandlungsoption bei finalen \\ Lungenerkrankungen. Sowohl vor als auch nach LTX gibt es \\ große überlebensrelevante psychosoziale Belastungen und \\ Herausforderungen, die bisher noch nicht qualitativ und im \\ Längsschnitt untersucht wurden. \\ Patient 2016; DOI: 10.1007/s40271-016-0174-Z
}

In einer aktuellen Studie wurden daher 40 erwachsene Patienten (davon 55\% männlich) nach LTX nach 2 Wochen (T1), 3 Monaten (T2) und 6 Monaten (T3) zur Häufigkeit von verschiedenen Gedanken, Gefühlen und Einstellungen zur LTX sowie der medikamentösen Behandlung interviewt. Im Mittel standen Patienten 9 Monate auf der Warteliste und waren nach LTX durchschnittlich 4,5 Wochen im Krankenhaus. Die 6-Monats-Überlebensrate lag in der Studie bei $100 \%$. Die Patienten berichteten von massiven körperlichen Verbesserungen (Bewegung ohne externe Sauerstoffzufuhr, keine Atemnot mehr, Sport treiben können), die mit einer Erleichterung und steigenden Lebenszufriedenheit einhergingen. Sich wieder um die eigene Familie kümmern zu können, trug besonders zum verbesserten emotionalen Befinden bei.

\section{Hilfreich trotz körperlicher und emotionaler Beschwerden $\nabla$}

Dennoch gab es weiterhin eine Vielzahl belastender körperlicher (Schmerzen, Fatigue, wiederkehrende Infektionen) und psychischer Beschwerden (Überforderungs- und Hilflosigkeitserleben, Sorgen hinsichtlich Organversagen und Abstoßungsreaktionen, Schlafstörungen, Depressivität und Ängstlichkeit), insbesondere aufgrund des langwierigen Heilungsprozesses, unerwünschter medikamentöser Nebenwirkungen (UEW), wiederholter Krankenhausaufenthalte und Unsicherheiten im Umgang mit der Medikation und auftretenden Symptomen. Die UEW zu T1 umfassten v.a. gastrointestinale Beschwerden (Übelkeit/Erbrechen), später Gewichtsveränderungen und neurologische Beschwerden (Tremor). Die medikamentöse Behandlung (Umfang, UEW) wurde als einschränkend im Alltag und organisatorische Herausforderung erlebt. Nur etwa $40 \%$ der Patienten beurteilten ihre medikamentöse Adhärenz als gut. Körperliche Beschwerden zu T1 und T2 waren mit vermehrtem emotionalem Distress zu T2 und T3 assoziiert.

\section{Fazit}

Körperliche Beschwerden belasten Patienten nach LTX besonders häufig und werden zeitlich von höheren emotionalen Belastungen gefolgt. Etwa 30\% der Patienten berichteten von klinisch relevanten emotionalen Schwierigkeiten durch die LTX (Unsicherheit und Angst hinsichtlich Erkrankungsverlauf und Behandlungsregime, Schuldgefühle gegenüber Spender). Hierzu scheint Handlungsbedarf auf Seiten des Behandlungsteams (Patientenschulungen, vermehrte Unterstützung beim Übergang ins häusliche Umfeld/Selbstmanagement) zu bestehen. Mit Ausnahme des Einschränkungserlebens im Alltag (deutlicher Anstieg von T1 [43\%] zu T2 [70\%]) persistiert das Belastungserleben der Patienten inhaltlich über die Zeit und scheint so den langwierigen Anpassungsprozess an das Transplantat und Transplantationserleben abzubilden. Die Transplantation wurde von Patienten mehrheitlich als rational-mechanischer Prozess erlebt. Von Integrationsprobleme des Organs in das eigene Körpererleben berichteten die wenigsten Patienten.

\section{Dr. Sarah Weusthoff, Hannover}

\section{Personalisierte Antibiotika-Therapie}

Ein Freiburger Forschungsteam hat ein von der Biologie inspiriertes Verfahren entwickelt, das verschiedene Antibiotika in menschlichem Blut oder in anderen Flüssigkeiten gleichzeitig nachweisen kann. Zukünftig ließe sich das BiosensorSystem in der medizinischen Diagnostik und besonders in sog. patientennahen Tests in Arztpraxen, Apotheken oder bei Hausbesuchen, aber auch in der Umweltund Lebensmittelkontrolle einsetzen.

Die Forschenden haben dazu menschliches Blut auf die Antibiotika Tetrazyklin und Streptogramin untersucht. „Die Analysezeit, von der Probenentnahme bis zum Ergebnis, liegt bei nur 10 Minuten“, erläutert der Mikrosystemtechniker Dr. Can Dincer, der das Team leitet. „In unserer Studie haben wir die Anwendbarkeit der Plattform demonstriert." Ihre Ergebnisse haben die Wissenschaftler in der Fachzeitschrift Analytical Chemistry (Anal Chem 2016; 88: 10036-10043) veröffentlicht.

In der Arbeitsgruppe von Prof. Gerald Urban wurde die elektrochemische Biosensor-Plattform, die mit kleinsten Flüssigkeitsmengen arbeitet, entwickelt. „Der größte Vorteil des Systems ist, dass wir bis zu 8 verschiedene Substanzen gleichzeitig, einfach und schnell messen können“, erklärt Dincer. Die Forschenden haben die Chip-Technologie mit einer Methode kombiniert, die die Arbeitsgruppe des Freiburger Biotechnologen Prof. Dr. Wilfried Weber mitentwickelt hat. Das Verfahren basiert auf dem natürlichen Sensor-Protein, mit dem resistente Bakterien die Medikamente wahrnehmen und daraufhin ihre Mechanismen aktivieren. Die bakteriellen Sensoren reagieren schnell, sensitiv und spezifisch auf Antibiotika und sind dadurch optimal für einen Einsatz in einem analytischen Test geeignet. So liefern die Bakterien den Forschenden ein Werkzeug, das sie langfristig bekämpfen soll.

Nach einer Mitteilung der Albert-LudwigsUniversität Freiburg 